\title{
Refractory, missed and severe Kawasaski disease: Diagnostic and therapeutic challenges
}

George Comitis*, John Lawrenson", Adre Van Schalkwyk ${ }^{\dagger}$ and Liesl Zühlke,";

*Paediatric Cardiology Service of the Western Cape, Red Cross Children's Hospital, Cape Town, Division of Critical Care and Children's Heart Diseases, School of Child and Adolescent Health, University of Cape Town, South Africa

\#Paediatric Cardiology Service of the Western Cape, Red Cross and Tygerberg Children's Hospitals, Department of Paediatrics and Child Health, University of Stellenbosch, South Africa

†Milnerton Mediclinic, Cape Town, South Africa

tDepartment of Medicine, Groote Schuur Hospital and

University of Cape Town, Cape Town, South Africa.

Address for correspondence:

Dr George A. Comitis

Division of Critical Care and Children's Heart Disease

School of Child and Adolescent Health

University of Cape Town

Red Cross Children's Hospital

Klipfontein Road

Rondebosch

7700

South Africa

Email:

george.comitis@uct.ac.za

\section{INTRODUCTION}

Kawasaki disease $(K D)$ is the most common acute vasculitis of childhood worldwide, affecting predominantly infants and young children with $80 \%$ of cases occurring below 5 years of age. It is currently the leading cause of acquired heart disease in children in the developed world.(')

The cause of KD remains unknown but is thought to be due to an abnormal immune response to an infectious trigger in a genetically predisposed individual.

Coronary artery abnormalities (CAA) are the main complication of $\mathrm{KD}$ and account for the majority of morbidity and mortality (mainly due to myocardial infarction secondary to acute thrombosis within coronary artery aneurysms). Current treatment regimens

\section{ABSTRACT}

Kawasaki disease (KD) is an acute generalised vasculitis of childhood with a predilection for the coronary arteries causing ectasia and aneurysms with subsequent potential ischaemic heart disease and even sudden death. This risk is significantly reduced by early treatment with intravenous immunoglobulin together with a high dose of aspirin. However, the diagnosis is often difficult to make as many childhood illnesses may mimic KD. In addition, presentations may be atypical or incomplete.

We discuss a series of 3 recent cases to focus on the management of 3 potentially troublesome aspects of the disease namely: severe coronary vascular involvement, failure to recognise the disease and disease which is refractory to standard therapy. We will also provide a suggested treatment algorithm for refractory KD with reference to current and future research strategies. SAHeart 20 |3; 0:462-468

have however reduced the acute mortality rate from about $2 \%$ in the 1970s to less than $0.2 \%$. $^{(2)}$

Prompt recognition of the disease and early initiation of treatment with intravenous polyvalent immunoglobulin (IVIG) (within the first 10 days) results in significant reduction in the occurrence of CAA - from 15 - 25\% to about 5\%. ${ }^{(2)}$ About half to 2 thirds of these will regress within the first I - 2 years (especially the smaller aneurysms) but others will persist with dilatation, stenoses, tortuosity and thrombosis. In addition, despite IVIG, I\% still develop giant aneurysms (measuring $8 \mathrm{~mm}$ or more). Furthermore, affected coronaries do not dilate normally with exercise or coronary vasodilators (even if the lesions have regressed). ${ }^{(3,4)}$ These persistent coronary lesions have obvious medium and long-term implications in adolescent and adult life, for example with regard to participation in sports and potential premature atherosclerotic coronary artery disease. ${ }^{(3)}$

This article reports on a recent cluster of KD patients seen at 2 hospitals in Cape Town. Each case highlights a particular learning point or challenge in the diagnosis or management.

\section{CASE I - SEVERE KD}

A 15-month-old previously well boy was admitted with diarrhoea and vomiting, cough and fever which had persisted for 4 days. On examination he was lethargic with a temperature of $38.6^{\circ} \mathrm{C}$ and an 
erythematous rash on the trunk. Initial investigations showed a white cell count (WCC) of $11.8 \times 10^{9} / 1$ with $58 \%$ bands, platelet count of $360 \times 10^{9} / 1$ and CRP $197 \mathrm{mg} / \mathrm{l}$.

The diagnosis of KD was made on day 2 - 3 of admission as his fever continued (up to $39.6^{\circ} \mathrm{C}$ ), he was irritable and developed all 5 principal clinical features, viz. non-exudative conjunctivitis, erythematous rash on the trunk and face, oedema of the hands and feet, unilateral cervical lymphadenopathy and cracked lips with a red tongue.

At this stage (day 6 of fever) he received IVIG $2 \mathrm{~g} / \mathrm{kg}$ plus aspirin at moderate dose $(40 \mathrm{mg} / \mathrm{kg} / \mathrm{d})$. His temperature settled over the next 4 days but recurred on day 8 of admission /day 12 of illness $\left(38.3^{\circ} \mathrm{C}\right)$.

An echocardiogram at this stage rather concerningly showed pancoronary dilatation as follows ( $Z$ scores ${ }^{(5)}$ in brackets): left main coronary artery (LMCA) 4.3mm (+6.4), distal left anterior descending coronary artery (LAD) $5 \mathrm{~mm}(+10.3)$, proximal circumflex coronary artery (Cx) $4 \mathrm{~mm}(+7.0)$, right coronary artery (RCA) $3.5 \mathrm{~mm}(+4.8)$.

Significantly there was a proximal LAD fusiform aneurysm of $5.6 \mathrm{~mm}$ and distal Cx saccular aneurysm of $6.1 \mathrm{~mm}$. Ventricular function was normal.

Based on these findings and the recurrence of fever, as well as rising inflammatory markers (WCC $22 \times 10^{9} / \mathrm{l}$ and CRP $322 \mathrm{mg} / \mathrm{l}$ ) he received a second dose of $I V I G 2 g / k g$ and his aspirin dose was increased to $90 \mathrm{mg} / \mathrm{kg} / \mathrm{d}$. Thereafter his temperature settled and his clinical features steadily resolved. He also received pulsed steroids (methylprednisolone $30 \mathrm{mg} / \mathrm{kg} / \mathrm{d}$ ) for 3 days during convalescence due to an isolated low grade fever of $37.7^{\circ} \mathrm{C}$, a persistently raised CRP of $57 \mathrm{mg} / \mathrm{l}$ and ESR of $90 \mathrm{~mm} / \mathrm{hr}$ as well as painful arthritis of his ankles and knees with effusions.

An echocardiogram on day 19 of illness showed an LAD fusiform giant aneurysm of $8-9 \mathrm{~mm}$ with suspicion of intraluminal thrombus (Figure I), distal Cx saccular aneurysm, dilated proximal RCA with a $5.5 \mathrm{~mm}$ saccular aneurysm in mid-vessel and normal LV function.

He was then started on low molecular weight heparin for 2 weeks and maintenance warfarin and his aspirin dose was reduced to $3 \mathrm{mg} / \mathrm{kg} / \mathrm{d}$. At 3 months an echocardiogram still showed aneurysms of the LAD, Cx and RCA. He remains asymptomatic.

\section{Practice point}

Cases of KD with this degree of coronary involvement (large aneurysms of all 3 major coronaries) have rarely been reported in the literature. ${ }^{(6)}$ The need for anti-coagulation of such (often young) patients (as prophylaxis against coronary thrombosis and myocardial infarction) adds a further element of complexity to their management. Another important consideration in such patients is adequate and accurate coronary imaging - although transthoracic echocardiography remains the initial modality of choice, more definitive imaging with transoesophageal echocardiography, computed tomographic angiography, magnetic resonance angiography or conventional coronary angiography is usually necessary to accurately delineate the lesions, especially to assess if revascularisation is indicated. The present patient is awaiting conventional selective coronary angiography.

In addition, this case highlights the fact that severe CAA can still occur despite prompt, appropriate therapy with the current standard regimen of IVIG plus aspirin (see Figure 6). This example (and the following case) is in keeping with the fact that young infants with $\mathrm{KD}$ have the highest incidence of coronary artery aneurysms.

The recently published RAISE study ${ }^{(7)}$ demonstrated reduced CAA in Japanese children with severe KD (defined using a validated risk score) ${ }^{(8)}$ treated with adjuvant corticosteroids in addition to the standard regimen. However, the difficulty lies in identifying early which children are likely to be IVIG-resistant (and hence at a greater risk of developing (AA) in the South African context since these children would likely benefit from adjunctive corticosteroids. The Japanese scoring system has low sensitivity in North American populations ${ }^{(9)}$ so may equally not be applicable in our setting.

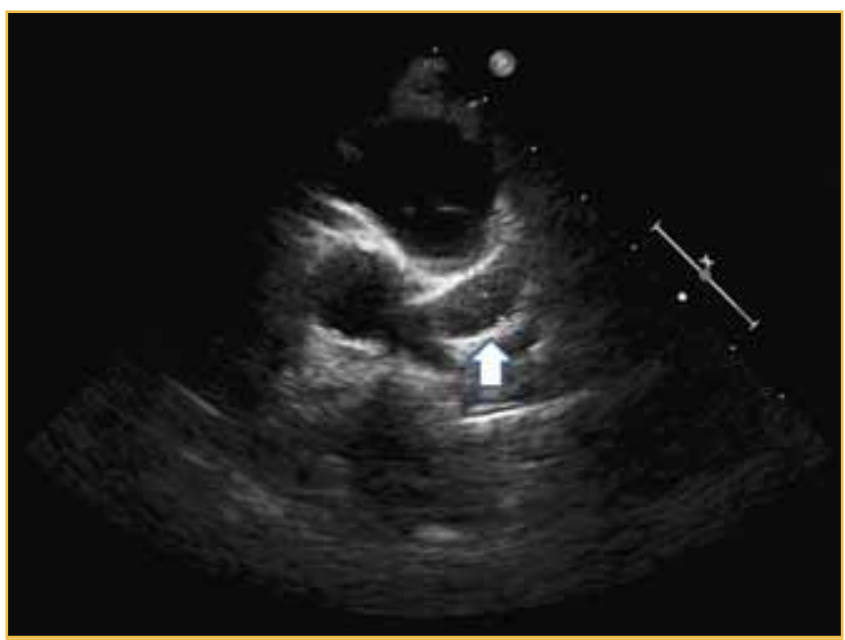

FIGURE I: Parasternal short axis view of LAD fusiform giant aneurysm in Case I 


\section{CASE 2 - MISSED KD}

A 6-year-old boy was referred with a I week with a history of tiredness, loss of appetite, fast breathing and rapid heart pulsations. At I year of age he had a severe febrile illness associated with an erythematous rash and red lips which required admission and intravenous antibiotics for I week at another institution.

On examination, he appeared chronically ill with wasting. He was not shocked but his pulses were thready and he had obvious features of heart failure. He responded to conventional heart failure treatment with furosemide, spironolactone, captopril, carvedilol as well as low dose aspirin.

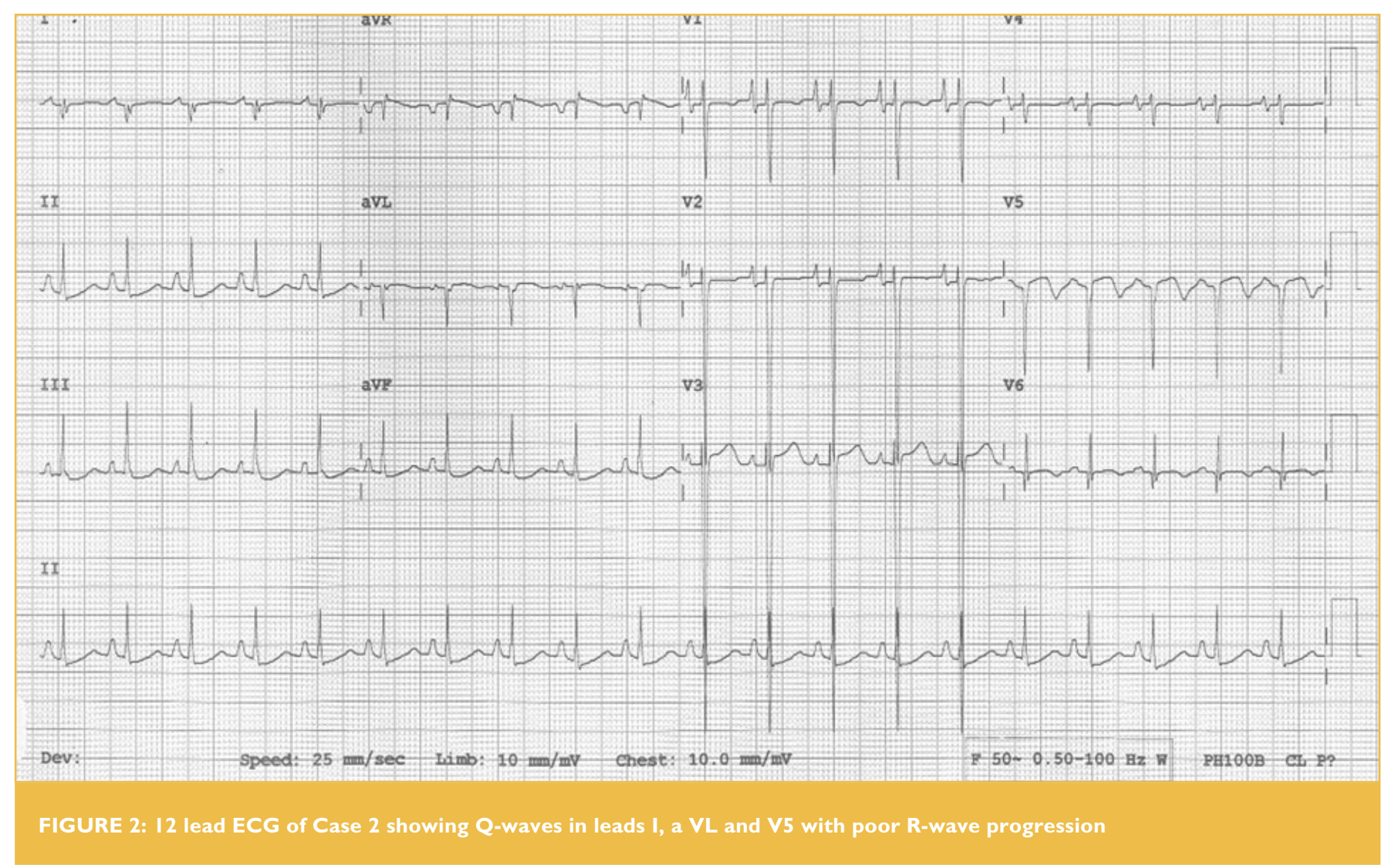

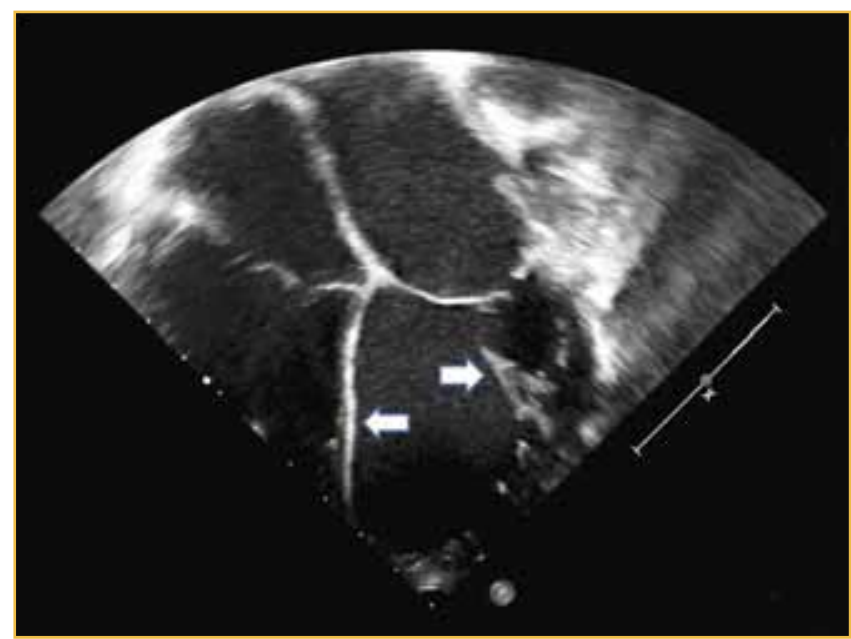

FIGURE 3: Apical 4 chamber view in Case 2 showing a dilated left heart and echobright IVS and LV papillary muscles

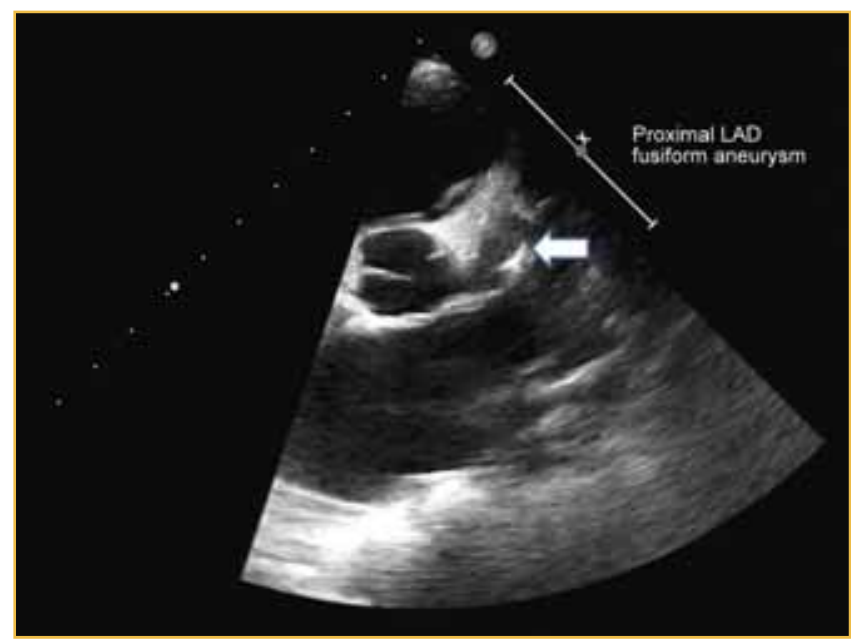

FIGURE 4: Parasternal short axis view of proximal LAD fusiform aneurysm in Case 2 
His ECG showed Q-waves in leads I, AVL and V5 with poor R-wave progression (Figure 2).

An echocardiogram revealed severely impaired LV systolic and diastolic function, and echobright LV papillary muscles and interventricular septum IVS suggesting infarcts in the LAD and RCA territories (Figure 3). There were obvious regional wall motion abnormalities with akinesia and poor thickening of the entire IVS and mid to apical posterior and lateral walls (only basal segments contracting normally). In addition there was a suspicion of an apical LV thrombus. His coronaries were dilated as follows ( $Z$ scores (5) in brackets): LMCA $4.6 \mathrm{~mm}(+6.2), C \times 5.0 \mathrm{~mm}(+8.6)$, distal LAD $4.5 \mathrm{~mm}(+7.8)$, distal RCA $2.5 \mathrm{~mm}(+2.5)$.

Significantly, there was a proximal LAD fusiform aneurysm of $7.7 \mathrm{~mm}$ (Figure 4) as well as proximal RCA aneurysm of $5.5 \mathrm{~mm}$ (Figure 5). As no other aetiology for his cardiomyopathy has been identified, the cause is presumed to be ischaemic in origin secondary to acquired CAA as above sustained during a missed episode of $K D$ at I year of age.

Subsequent coronary angiography revealed a long segment occlusion of the proximal LAD with some filling of the distal $L A D$ via tiny collaterals. Further imaging is planned to detect viable myocardium and reversible ischaemia (dobutamine stress echocardiogram and myocardial perfusion scan) to see if he will benefit from revascularisation.

\section{Practice point}

This case illustrates the significant morbidity that can accompany CAA secondary to KD and the potentially devastating effect of a missed diagnosis. Potentially many children with KD remain undiagnosed because of lack of awareness as well as a wide differential diagnosis such as viral infections (e.g. measles, adenovirus, enterovirus, Epstein-Barr virus), scarlet fever, staphylococcal scalded skin and toxic shock syndromes and drug reactions including StevensJohnson syndrome.

In addition 15\% of children present with "incomplete" KD and CAA. These children do not fulfil the classic criteria but exhibit echocardiographic features of CAA. This is more common in young infants who are also at highest risk of CAA. Hence referral for echocardiography should be considered in all children with unexplained fever for 5 or more days with or without any of the principal clinical features of KD especially in the presence of supportive laboratory evidence such as elevated ESR, CRP, liver enzymes and bilirubin, low albumin, sterile pyuria and aseptic meningitis. ${ }^{(2)}$

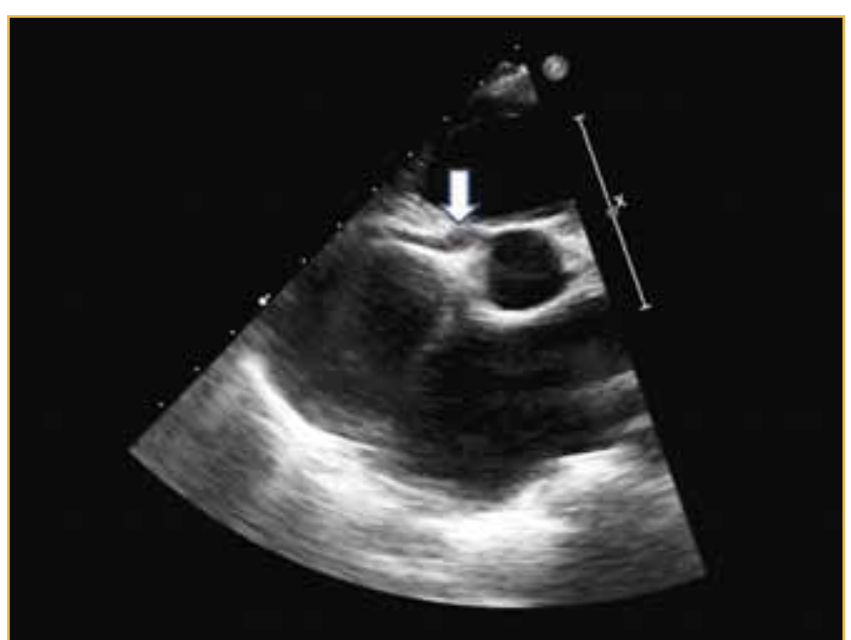

FIGURE 5: Parasternal short axis view of proximal RCA saccular aneurysm in Case 2

\section{CASE 3 - REFRACTORY KD}

A 17-month-old boy was admitted to a local institution with an upper respiratory tract infection. He had a two day history of high fever, nasal congestion and cough. He was febrile, had evidence of non-exudative pharyngeal and tonsillar inflammation and nontender cervical lymphadenopathy. Initial treatment included antipyretics, oral antibiotics, topical antiseptic and anti-inflammatory spray. Over the following 24 hours, he deteriorated rapidly developing persistent high fevers, a spreading erythematous polymorphous rash with hyperaemic swollen hands and feet and clinical meningism. His lumbar puncture was within normal limits. The CRP was raised at 53.6mg/l, his WCC was $25.8 \times 10^{\circ} / 1$ and he had a normochromic normocytic anaemia. Intravenous antibiotics were commenced and the diagnosis of Kawasaki disease entertained. Within a further 24 hours, he developed a hepatomegaly, bilateral conjunctivitis and cracked, red lips. At this point, he was given an IVIG infusion $2 \mathrm{~g} / \mathrm{kg}$ and commenced on high dose aspirin. His fever nevertheless continued to spike and his clinical condition worsened necessitating ICU admission. He developed a large hepatomegaly, rapid desquamation, severe conjunctivitis and abdominal distention associated with severe oedema. The dose of IVIG was repeated without any improvement. An echocardiogram revealed dilated left and right coronaries each measuring 2 - 3mm. He continued to deteriorate with rapidly increasing inflammatory markers (CRP $334 \mathrm{mg} / \mathrm{l}, \mathrm{ESR}>150 \mathrm{~mm} / \mathrm{hr}$ ). He was thus given a dose of methylprednisolone of $10 \mathrm{mg} / \mathrm{kg}$ over 4 hours. This resulted in significant hypertension of $160 / 120 \mathrm{mmHg}$ with the result that doses 2 and 3 were administered over 6 hours. Although the blood pressure stabilised, the pyrexia continued and the patient remained extremely unwell, with progression of inflammatory activity evident. A repeat echocardiogram at this point showed further dilatation of both left main coronary and circumflex arteries up to $4-5 \mathrm{~mm}$ 
and progression in the dilatation of the right coronary now measuring $3 \mathrm{~mm}$. Finally on day 11, 9 days after admission and 6 days after the first dose of IVIG, an infliximab infusion was given at $5 \mathrm{mg} / \mathrm{kg}$ over several hours. By the following morning, the patient had improved dramatically, was afebrile for the first time since admission, able to eat, converse and play. His oedema rapidly resolved and within 72 hours, all his inflammatory markers had reduced and he was ready for discharge. A pre-discharge echocardiogram revealed fusiform dilatation of left and right coronary arteries both measuring $3-4 \mathrm{~mm}$. No aneurysms were noted. Currently the patient is well and thriving.

\section{DISCUSSION}

The mainstay of treatment of KD remains intravenous immunoglobulin (IVIG) in combination with high-dose aspirin followed by reduction in the aspirin dose following defervescence. ${ }^{(2)}$ However, as the incidence of KD increases in the developed world so too do reports of immunoglobulin resistance. ${ }^{(10)}$ Cases I and 3 above illustrate the problem of IVIG-resistant KD and raise the question of effective second-and third-line therapy.

Up to I $5 \%$ of patients do not respond to an initial IVIG dose - these patients are at a significantly greater risk of developing CAA and may require re-treatment or further anti-inflammatory therapy. (I) The central culprit in KD is activation of the inflammatory cascade with an outpouring of cytokines producing both fever and the potentially devastating inflammatory vasculitis resulting in coronary and peripheral artery damage. A large US/Canadian study showed that patients who remained febrile had an almost nine-fold increase in CAA compared to those who responded to IVIG. ${ }^{(12)}$ Thus it is critical to institute additional therapy in any child who remains febrile after treatment or re-treatment of KD with standard therapy.

Corticosteroids have been suggested as a next step after a repeat dose of IVIG for more than a decade. ${ }^{(13)}$ However, this has been controversial with some reports suggesting that corticosteroids may in fact be harmful when used in this context. ${ }^{(14)} \mathrm{A}$ meta-analysis reviewing regimens including corticosteroids in the primary treatment of Kawasaki disease revealed a significant reduction in retreatments with IVIG in patients receiving corticosteroid plus standard therapy compared to standard therapy alone.(15) There was however no significant reduction in the incidence of coronary artery aneurysms among patients who received corticosteroids plus standard therapy, compared to standard therapy alone. Recent data dispute these findings and suggest a significant beneficial effect of steroids. ${ }^{(16)}$ It has also recently been reported as an adjunctive therapy for patients with severe KD in a large multi-centre, prospective, randomised controlled trial (RAISE study). ${ }^{(7)}$ This showed that in Japanese children, where prednisolone was added to the standard regimen of IVIG, the intensified primary treatment group had a significantly lower incidence of CAA.

Adjuvant and experimental therapies for refractory $\mathrm{KD}$ include additional doses of IVIG, methotrexate, abciximab, ulinastatin, cyclophosphamide, plasmapheresis and tumor necrosis factor-a (TNF- $\alpha$ ) blockers such as infliximab. ${ }^{(17,18)}$ Infliximab is a monoclonal antibody against TNF alpha and as such carries potential side-effects of immunosuppression or immune reactions. The rationale behind its use relates to suppressing the pro-inflammatory cytokines such as TNF- $\alpha$, interleukins and endothelial growth factor that are elevated during the acute phase of KD. Increased serum levels of these correlate with coronary aneurysm and subsequent stricture formation. TNF- $\alpha$ blockade is now increasingly being used for KD patients with recrudescent fever or persistently elevated inflammatory markers to prevent the endothelial damage which is thought to result in severe endothelial cell dysfunction and apoptosis. ${ }^{(19)}$ Clinical evidence to support the use of TNF- $\alpha$ blockade has been promising though anecdotal.(20,21) However in 2005, Burns, et al. reported a larger series of refractory KD patients treated using infliximab with excellent results. ${ }^{(22)}$ More recently, these authors reported a randomised prospective trial that demonstrated the safety, tolerability and pharmacokinetics of TNF- $\alpha$ blockade versus a second dose of IVIG. ${ }^{(23)}$ Importantly, studies of anti-TNF- $\alpha$ have not demonstrated increased side effects from drug administration such as infusion reactions, reactivation of infectious diseases (in particular tuberculosis), bacterial sepsis or increased risk of lymphoma. It is clear that further investigation of the role of infliximab in IVIGresistant patients is needed, especially as recognition of this condition improves and more patients are diagnosed timeously. ${ }^{(24)}$ Results are awaited from a recently completed large multi-centre study in the USA comparing standard therapy with standard therapy plus infliximab as initial therapy for all patients with KD. ${ }^{(25)}$

Patients not responding to these agents may be considered for other TNF- $\alpha$ blockade therapy. Etanercept (Enbrel) is another TNF- $\alpha$ inhibitor that has been studied in a few KD patients. Thus far it has shown some efficacy and is also under investigation as adjuvant therapy. ${ }^{(26)}$ The calcineurin inhibitor, cyclosporine A (CSA) has long been used as a potent immunosuppressive in transplantation. A new role has been suggested for CSA in refractory KD. This follows evidence of the role that $T$ cells and calcineurin/nuclear factor of the activated T cell NFAT pathway play in KD. As CSA is a potent inhibitor of $T$ cells via inhibition of the same NFAT pathway, this strategy seems opportune. A recent publication has detailed a protocol using CSA in IVIG -resistant KD. ${ }^{(27,28)}$ Other therapies for refractory KD have been limited to case series and reports and include successes with methotrexate, plasma exchange and ulinastatin, a trypsin inhibitor. However, these therapies, though promising, need further study. ${ }^{(29)}$ 


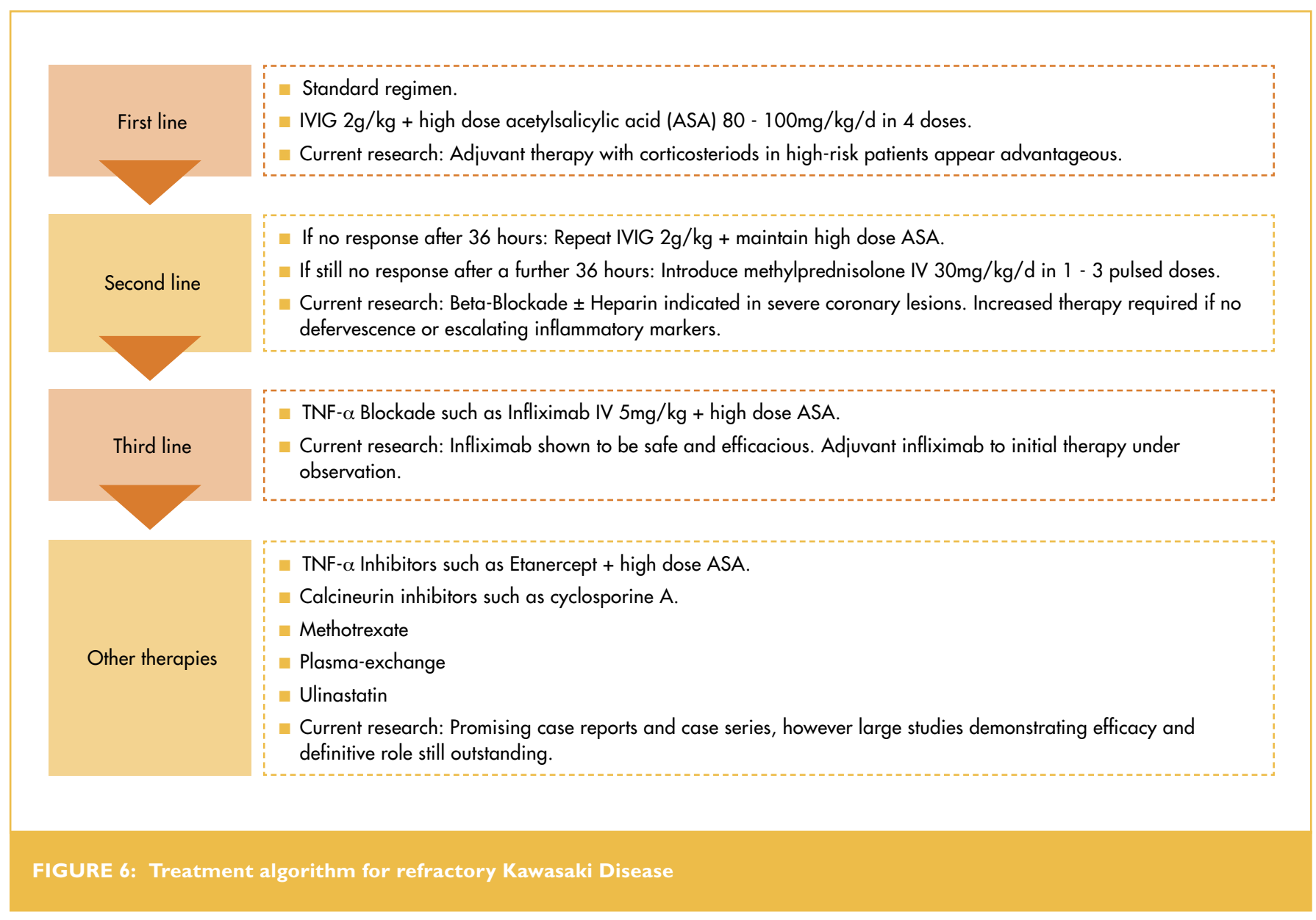

In summary, repeat IVIG remains the initial second-line therapy for continued fever followed by corticosteroids. In cases refractory to both these therapies, infliximab is the most commonly used thirdline agent.

Figure 6 summarises the current recommendations for initial therapy and outlines options for second and third-line treatment. As more KD patients are encountered, the importance of considering these treatment options must be stressed if 2 doses of IVIG do not result in the desired defervescence and reduction in inflammatory markers.

\section{CONCLUSION}

Kawasaki disease is responsible for significant cardiovascular morbidity with often permanent sequelae and potential major impact on cardiovascular health in later life. This can be minimised with improved recognition of the condition together with awareness of incomplete presentations amongst general practitioners and paediatricians followed by prompt and appropriate therapy to reduce the incidence of potentially devastating CAA. Further improvements in outcomes may come from early recognition of children at greatest risk for IVIG-resistance and development of CAA in order to provide adjunctive corticosteroids to the primary treatment (which are relatively cheap and therefore promise to be the most effective approach in developing countries) as well as other potential primary therapies such as TNF- $\alpha$ blockade to prevent the development of coronary complications.

\section{ACKNOWLEDGMENTS}

To the parents of our patients, who graciously allowed us to use their children's cases to illustrate important clinical lessons, our thanks and appreciation.

Drs Liesel Andrag and Rik De Decker for images for Case 2.

Drs Rik De Decker, Harold Pribut and Chris Scott for helpful comments and editing of this manuscript.

\section{FUNDING STATEMENT}

Liesl Zühlke is funded by the Thrasher Foundation and the Hamilton Naki Clinical Scholarship Programme funded by Netcare Limited.

\section{Conflict of interest: none declared.}




\section{REFERENCES}

I. Baker AL, Newburger JW. Cardiology patient pages. Kawasaki disease. Circulation. 2008 Aug 12; I 8(7):el 10-2.

2. Newburger JW, Takahashi M, Gerber MA, Gewitz MH, Tani LY, Burns JC, et al. Diagnosis, treatment, and long-term management of Kawasaki disease: A statement for health professionals from the committee on rheumatic fever, endocarditis, and Kawasaki disease, council on cardiovascular disease in the young, American Heart Association. Circulation. 2004; 1 10:2747-277।.

3. lemura $M$, Ishii $M$, Sugimura $T$, Akagi $T$, Kato $H$. Long term consequences of regressed coronary aneurysms after Kawasaki disease: Vascular wallmorphology and function. Heart. 2000;83:307-3II.

4. Sugimura $T$, Kato $H$, Inoue $O$, Takagi J, Fukuda $T$, Sato N. Vasodilatory response of the coronary arteries after Kawasaki disease: Evaluation by intracoronary injection of isosorbide dinitrate. Journal of Paediatrics. 1992; 121:684-688.

5. Dallaire F, Dahdah N. New equations and a critical appraisal of coronary artery $Z$ scores in healthy children. Journal of the American Society of echocardiography. 2011 Jan;24(1):60-74.

6. Gupta P, Gulati GS, Kothari SS. Kawasaki disease: A rare case of diffuse coronary involvement. Paediatric cardiology 2012;33:1218-1219.

7. Kobayashi T, Saji T, Otani T, Takeuchi K, Nakamura T, Arakawa H, et al. Efficacy of immunoglobulin plus prednisolone for prevention of coronary artery abnormalities in severe Kawasaki disease (RAISE study): A randomised, open-label, blinded-endpoints trial. Lancet. 2012 Apr 28;379(9826): 1613-20.

8. Kobayashi T, Inoue $\mathrm{Y}$, Takeuchi $\mathrm{K}$, et al. Prediction of intravenous immunoglobulin unresponsiveness in patients with Kawasaki disease. Circulation 2006; I13: 2606-12.

9. Newburger JW. Adding prednisolone to standard immunoglobulin therapy is beneficial for patients with severe Kawasaki disease. Journal of Paediatrics. 2012 Nov; I6I (5):967.

10. Tremoulet AH, Best BM, Song S, Wang S, Corinaldesi E, Eichenfield JR, et al. Resistance to intravenous immunoglobulin in children with Kawasaki disease. Journal of Paediatrics. 2008 Jul; | 53(1): I 17-21.

II. Wallace CA, French JW, Kahn SJ, Sherry DD. Initial intravenous gammaglobulin treatment failure in Kawasaki disease. Paediatrics. 2000 Jun; I05(6):E78.

12. Burns JC, Capparelli EV, Brown JA, Newburger JW, Glode MP. Intravenous gamma-globulin treatment and retreatment in Kawasaki disease. US/Canadian Kawasaki syndrome study group. The Paediatric infectious disease journal. Comparative study. 1998 Dec; 17(12):1 |44-8

13. Al-Mayouf SM. The use of corticosteroid therapy in refractory Kawasaki patients. Clinical rheumatology. 2004 Feb;23( I): I 1-3.

14. Kato H, Koike S, Yokoyama T. Kawasaki disease: effect of treatment on coronary artery involvement. Paediatrics. 1979 Feb;63(2): 175-9.

15. Athappan G, Gale S, Ponniah T. Corticosteroid therapy for primary treatment of Kawasaki disease - weight of evidence: a meta-analysis and systematic review of the literature. Cardiovascular Journal of Africa. 2009 Jul-Aug;20(4):233-6.

16. Son MB, Newburger JW. Management of Kawasaki disease: corticosteroids revisited. Lancet. 2012 Apr 28;379(9826):I57|-2

17. Blaisdell LL, Hayman JA, Moran AM. Infliximab treatment for paediatric refractory Kawasaki disease. Paediatric cardiology. 201 I Oct;32(7): 1023-7.

18. Song MS, Lee SB, Sohn S, Oh JH, Yoon KL, Han JW, et al. Infliximab treatment for refractory Kawasaki disease in Korean children. Korean Circulation Journal. 2010 Jul;40(7):334-8

19. Oishi T, Fujieda M, Shiraishi T, Ono M, Inoue K, Takahashi A, et al. Infliximab treatment for refractory Kawasaki disease with coronary artery aneurysm. Circulation Journal. 2008 May; 72(5):850-2

20. Girish M, Subramaniam G. Infliximab treatment in refractory Kawasaki syndrome. Indian Journal of Paediatrics. 2008 May;75(5):521-2.

21. Sivakumar K, Pavithran S. Extensive coronary aneurysms with thrombosis in resistant Kawasaki disease. Paediatric cardiology. 2013 Feb;34(2):444-6.
22. Burns JC, Mason WH, Hauger SB, Janai H, Bastian JF, Wohrley JD, et al. Infliximab treatment for refractory Kawasaki syndrome. Journal of Paediatrics. 2005 May: 146(5):662-7.

23. Burns JC, Best BM, Mejias A, Mahony L, Fixler DE, Jafri HS, et al. Infliximab treatment of intravenous immunoglobulin-resistant Kawasaki disease. Journal of Paediatrics. 2008 Dec; I53(6):833-8.

24. Weng KP, Ou SF, Lin CC, Hsieh KS. Recent advances in the treatment of Kawasaki disease. Journal of the Chinese Medical Association. 20I I Nov;74(I I):48 I-4.

25. Burns JC et al. Infliximab plus intravenous immunoglobulin (IVIG) for the primary treatment of patients with acute Kawasaki disease. Clinical trials online. Available from: http://clinicaltrials.gov/ct2/show/study/NCT00760435.

26. Portman MA, Olson A, Soriano B, Dahdah N, Williams R, Kirkpatrick E. Etanercept as adjunctive treatment for acute Kawasaki disease: study design and rationale. American Heart Journal. Randomised controlled trial research support, N.I.H., Extramural. 20I I Mar; I6I (3):494-9.

27. Suzuki H, Terai M, Hamada H, Honda T, Suenaga T, Takeuchi T, et al. Cyclosporin A treatment for Kawasaki disease refractory to initial and additional intravenous immunoglobulin. The Paediatric infectious disease journal. Research support, Non-U.S. Gov't. 20II Oct;30(I0):87I-6.

28. Tremoulet $A H$, Pancoast $P$, Franco A, Bujold $M$, Shimizu C, Onouchi $Y$, et al. Calcineurin inhibitor treatment of intravenous immunoglobulin-resistant Kawasaki disease. The Journal of Paediatrics. Research support, N.I.H., extramural research support, Non-U.S. Gov't. 2012 Sep; 161 (3):506- 12.

29. Dominguez SR, Anderson MS. Advances in the treatment of Kawasaki disease. Current opinion in paediatrics. 2013 Feb;25(I):103-9. 